\title{
The Chinese version of the American shoulder and elbow surgeons standardized shoulder assessment form questionnaire, patient self-report section: a cross-cultural adaptation and validation study
}

\author{
Tung-Hee Albert Tie ${ }^{1}$, Chih-Kai Hong ${ }^{2}$, Illich Chua ${ }^{3}$, Fa-Chuan Kuan ${ }^{2,4}$, Wei-Ren Su${ }^{2,5}$ and Kai-Lan Hsu 2,4 $^{*}$ (D)
}

\begin{abstract}
Background: The patient self-report section of the American Shoulder and Elbow Surgeons Standardized Shoulder Assessment Form (ASESP) is one of the most validated and reliable assessment tools. This study aimed to establish a validated Chinese version of ASESp (ASESp-CH).

Methods: A clinical prospective study was performed (ClinicalTrials.gov Identifier: NCT04755049; registered on 2021/ 02/11). Following the guidelines of forward-backward translation and cross-cultural adaptation, a Chinese version of ASESp was established. Patients older than 18 years with shoulder disorders were included. Patients who could not complete test-retest questionnaires within the interval of 7-30 days and patients who received interventions were excluded. Intraclass correlation (ICC) was calculated for test- retest reliability, whereas internal consistency was determined by Cronbach value. Construct validity was evaluated by comparing the corresponding domains between the ASESp-CH and a validated Chinese version of 36-Item Short Form Health Survey (SF-36).

Results: A total of 86 patients were included with a mean test-retest interval of $12 \pm 5.4$ days. Test-retest reliability was excellent with an ICC of 0.94. Good internal consistency was found, with a Cronbach alpha of 0.86. Construct validity of the ASESp-CH questionnaire was good. The major domains of the ASESp-CH were significantly correlated with the respective domains in the SF-36 ( $p<0.01$ ), except for the domain of stability of ASESp-CH.
\end{abstract}

Conclusions: The Chinese version of ASESp questionnaire is a highly validated and reliable tool for shoulder disorder assessment.

Keywords: ASES score, Cross-cultural adaptation, Validation, Patient self-reported questionnaire

\footnotetext{
* Correspondence: dulendulen@gmail.com

${ }^{2}$ Department of Orthopaedic Surgery, National Cheng Kung University Hospital, College of Medicine, National Cheng Kung University, No.138, Sheng-Li Road, North Dist., Tainan City 70428, Taiwan

${ }^{4}$ Department of Biomedical Engineering, National Cheng Kung University, No.138, Sheng-Li Road, North Dist., Tainan City 70428, Taiwan

Full list of author information is available at the end of the article
}

(c) The Author(s). 2021 Open Access This article is licensed under a Creative Commons Attribution 4.0 International License, which permits use, sharing, adaptation, distribution and reproduction in any medium or format, as long as you give appropriate credit to the original author(s) and the source, provide a link to the Creative Commons licence, and indicate if changes were made. The images or other third party material in this article are included in the article's Creative Commons licence, unless indicated otherwise in a credit line to the material. If material is not included in the article's Creative Commons licence and your intended use is not permitted by statutory regulation or exceeds the permitted use, you will need to obtain permission directly from the copyright holder. To view a copy of this licence, visit http://creativecommons.org/licenses/by/4.0/. The Creative Commons Public Domain Dedication waiver (http://creativecommons.org/publicdomain/zero/1.0/) applies to the data made available in this article, unless otherwise stated in a credit line to the data. 


\section{Background}

Shoulder pain is one of the most common musculoskeletal disorders in the human population [1-3]. A shoulder pathology may lead to pain, disability, and immobility which eventually affect one's activity of daily living (ADL) and quality of life (QOL) resulting in a socio-economic burden [2, 4, 5]. Similarly, elbow pain due to instability or consequences of traumatic events compromised one's QOL as well [6]. To reach a better prognosis in approaching shoulder and elbow disorders, functional improvement is an essential outcome. Apart from clinician-reported outcome instruments, patientreported outcome measures (PROMs) are recognized to be essential and increasingly used to quantify a patient's perceptions of functional ability in recent decades $[4,7]$. There are several PROMs available to evaluate a patient's condition, the patient self-evaluation section of the American Shoulder and Elbow Surgeons Standardized Shoulder Assessment Form Questionnaire (ASESp), one of the most widely used PROMS at the present time, is a highly validated and reliable PROM compare to the similar items $[4,5]$. It is easy and quick to use this validated questionnaire for evaluating shoulder problems $[8,9]$.

Due to diversity in the culture and natives' languages in different regions, a cross-cultural adaptation of ASESp shall be established. Currently, the ASESp questionnaire has been translated and validated into different languages to accommodate the clinical practices and research-needs including Dutch, Italian, Finnish, Spanish, German, Portuguese, Turkish, Arabic and Argentina [4, 8, 10-15].

The language is an indirect indicator of culture, an incorrect interpretation due to a non-validated translated questionnaire could lead to differences in functional score obtained and thus, low credibility [4]. Apart from minimizing the language barrier, having a validated scale in the local language is beneficial to clinical practice and future research in Chinese speaking countries. Currently, several Chinese versions of ASESp were attainable on the Internet; however, a unified and validated version has yet to be found. The purpose of this study was to conduct the translation and establish a cross-cultural adapted and validated Chinese version of the ASESp score (ASESp-CH).

\section{Methods}

The present study was officially authorized by the American Shoulder and Elbow Surgeons Society, the original developer of ASESp. Informed consents were obtained from all participants prior to the study.

\section{ASES questionnaires}

ASES composed of 3 sections: demographic information, patient self-evaluation (ASESp), and physician assessment [8]. A clinician is responsible to provide his or her expertise to evaluate the range of motion, strength, instability and other shoulder pathology signs; however, a score index can only be derived from the ASESp section for a thorough assessment [14].

The ASESp consists of 18 questions from 3 sections: pain, instability, and activities of daily living (ADL). Among the 18 questions, 11 self-report items representing functional (ADL) dimension (10 items) and pain dimension ( 1 item) are derived into a $0-50$ sub-score for each dimension [14]. The ADL section was scored in a 4-points- graded ordinal scale, ranging from 0 (unable to do) to 3 (not difficult) and cumulative scores were collected. The pain section was derived from the 10-pointsgraded visual analog scale (VAS) ranging from 0 (no pain) to 10 (maximum pain) [7]. The overall shoulder score index was calculated with the formula below, ranging from 0 (most disability) to 100 (least disability) [8].

$$
\begin{aligned}
\text { Shoulder score index } & =[(10-\text { VAS pain score }) \times 5] \\
& +[(5 / 3) \times \text { cumulative ADL score }]
\end{aligned}
$$

\section{Translation and linguistic validation}

A 5 steps protocol of forward-backward translation was derived based on the Beaton's method published in the Guidelines for the Process of Cross-Cultural Adaptation of Self-Report Measures [16]. The protocol included translation, synthesis, back-translation, expert committee review and pretesting [16-20].

\section{Step 1: forward translation from English to Chinese}

A forward translation was done separately by two native Chinese native speakers, one acting as an informed translator (senior orthopedic resident) and one acting as an un-informed translator (medical student).

\section{Step 2: cross-culture adaptation}

Several cross-cultural dissimilarities were found in the first translated version. First, in question 4, the example of pain medications given in original ASES, namely aspirin, Tylenol, Advil, Codeine, were replaced with aspirin and acetaminophen together with the respective Chinese brand name. Similarly, the narcotic medication given in question 5 was also replaced with tramadol, Ultracet and morphine together with the respective Chinese brand name. These modifications were done based on the most common, most familiar and typical prescription in Taiwan. Secondly, we clearly define the description of "Manage Toileting" in question 4 of the ADL section as a "butt-wiping" situation. Third, the "10 lbs" in question 7 of the ADL section was translated and remarked as " 5 kg" as kilogram, which was a typical measurement unit in Taiwan. 
Step 3: backward translation from Chinese to English

A backward translation was done by an English native speaker who was not familiar with the orthopedics field after a Chinese consensus version was completed.

\section{Step 4: revision by expert committees}

Both forward and backward versions were then revised and reviewed by an expert committee, which composed of five senior orthopaedic surgeons, including the chief of department of orthopaedic surgery. Both versions revealed no marked disparity or language difficulties. Thus, the primary Chinese ASES questionnaire (ASESp-CH) was formed.

\section{Step 5: pre-test of ASESp-CH questionnaires}

The ASESp-CH questionnaire was given to 20 patients to disclose any problem in understanding and approaching the questionnaire. There were no obstacles reported. Hence, a final ASESp-CH questionnaire was established (Fig. 1).

\section{Study population}

The study was conducted by the Department of Orthopedic Surgery in National Cheng Kung University Hospital (NCKUH) in Tainan, Taiwan, and was approved by the Institutional Review Board of National Cheng Kung
University Hospital. Patients with shoulder disorder were recruited from the out-patient department of NCKUH and the public population. All patients were required to complete the test-retest ASESp-CH questionnaires twice at the interval of 7 days to 30 days before getting any intervention. A questionnaire of SF-36 was also required to complete during the retest session of ASESp-CH. A thorough explanation and informed consent were given.

Inclusion criteria were as follow: [1] patients' age $\geq 18$ years, [2] patients with clear insights, [3] patients with any shoulder disorders, [4] patients who are able to speak and write in Chinese, and [5] patients who completed the questionnaires twice at an interval of 7 days to 30 days. Patients with one of the following conditions were excluded: [1] the patient could not complete all of ASESp-CH and SF-36 questionnaires, [2] the test-retest interval was less than 7 days or more than 30 days, and [3] the patient received interventional procedures, such as shoulder injections or surgery, during the test-retest interval.

\section{Reliability}

Reliability was considered as the degree of replicable, is the extent to which the results can be reproduced when the research is repeated under the same conditions. In this case, refers to the degree of the results of ASESp-

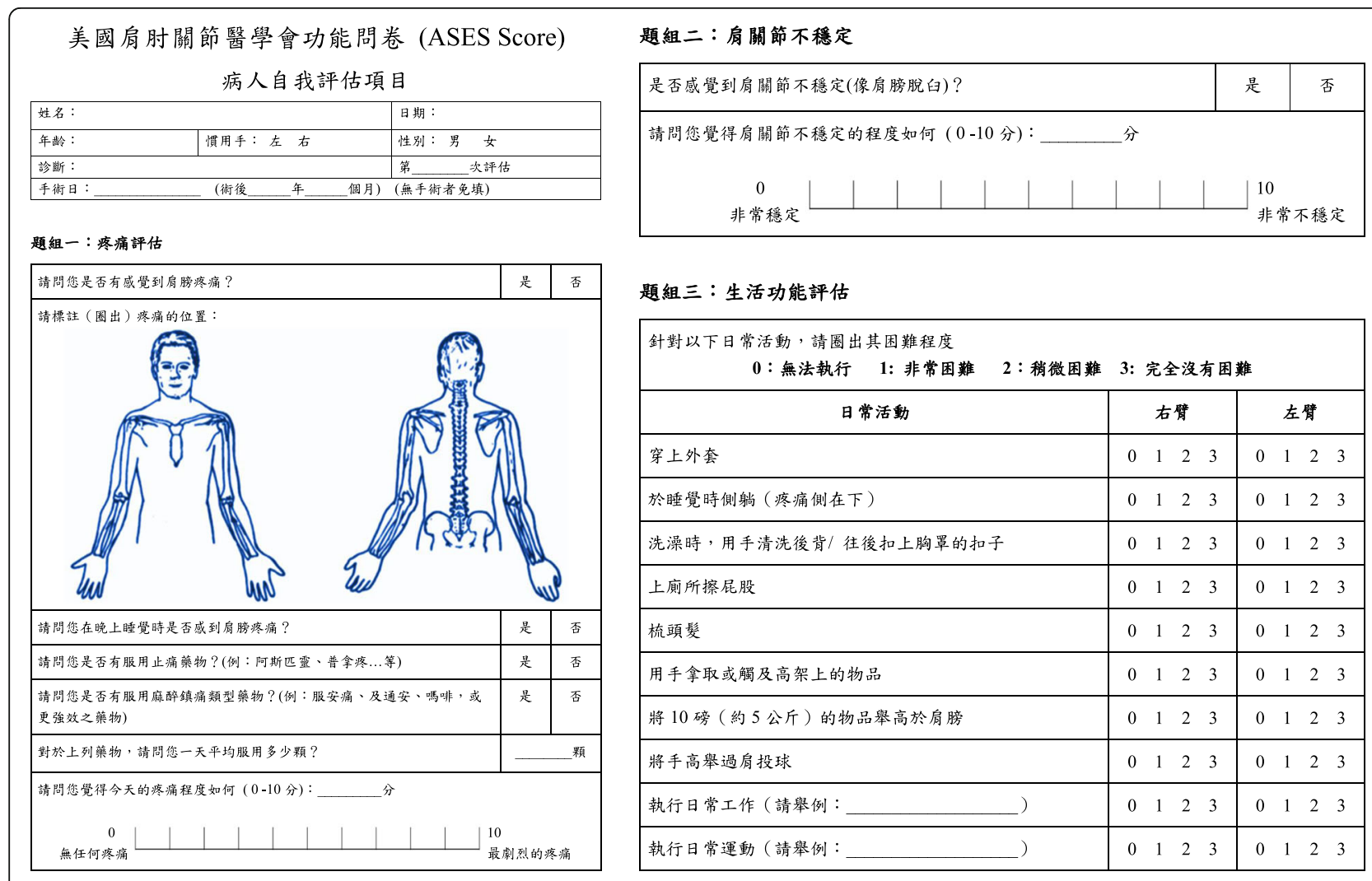

Fig. 1 Chinese version of the American Shoulder and Elbow Surgeons Standardized Shoulder Assessment Form (ASESp-CH) 
$\mathrm{CH}$ can be replicated in a test-retest manner and among related items on the ASESp-CH (internal consistency) [8]. Reliability can be expressed ranging from 0 to 1 , indicates no reliability and absolute reliability respectively.

Internal consistency is calculated by using the Cronbach alpha, a widely recognized analysis tool to evaluate one's reliability [21]. This method has been used in previous ASESp validation studies [4, 8, 10-15]. A cut-off value of 0.7 represents a sufficient correlation between the items of a questionnaire. Values between 0.7 to 0.79 , 0.80 to 0.89 and above 0.90 imply fair, good and excellent respectively. Yet, a Cronbach alpha greater than 0.90 may indicate a highly homogenous situation and thus redundant [8].

While considering the test-retest reliability, it was assumed that 2 separate measurements in a suitable interval should be similar if no change occurs and the timebias can be reduced to the minimum level. A short interval may cause memory bias, while a longer interval may encounter actual changes in patient health status [8]. Thus, a time interval between 7 to 30 days was considered relevant according to the guidelines of crosscultural adaptation. To calculate the test-retest reliability, the intraclass correlation coefficient (ICC) was used $[8,22]$. An ICC of 0 indicated no agreement, whereas an ICC of 1 indicated absolute agreement. Generally, an ICC greater than 0.60 and 0.74 were considered good and excellent, respectively [22].

\section{Validity}

To achieve the validation of ASESp-CH, the results collected from 3 domains of pain, instability, and ADL are compare with the corresponding 8 domains of 36-Item Short Form Survey (SF-36) [23]. As a widely accepted and validated health status measure, SF-36 also has been used as a parameter for the various translated versions of ASESp in the previous studies [4, 8, 10-14]. A translated and validated Taiwan version of SF-36 was eligible for the validation process $[24,25]$.

Pearson correlation coefficient was used to evaluate the construct validity between the 3 domains of ASESp$\mathrm{CH}$ and the corresponding domains in SF-36. Statistical analysis was performed using SPSS 20.0 (IBM, Armonk, NY, USA) and Excel 2010 (Microsoft, Redmond, WA, USA). A $p<0.05$ was considered statistically significant.

\section{Results}

In this study, a total of 112 patients with shoulder disorders were asked to participate from March 1st, 2020 to September 30th, 2020. Sixteen patients who refused to participate and 10 patients who did not complete the required questionnaires were excluded. Finally, 86 of the patients were included and the patients' demographic data were shown in Table 1.
Table 1 Patient characteristics $(n=86)$

\begin{tabular}{ll}
\hline & No. (\%) \\
\hline Sex & \\
Age, year, mean (SD) & $30(34.9)$ \\
Test-retest interval, days, mean (SD) & $39.2( \pm 17.6)$ \\
Affected side & $12.4( \pm 5.3)$ \\
Right shoulder & \\
Left shoulder & $54(62.8)$ \\
Bilateral shoulder & $28(32.6)$ \\
Diagnosis & $4(4.7)$ \\
Rotator cuff tear & \\
Frozen shoulder & $25(29.1)$ \\
Labrum lesions & $5(5.8)$ \\
Shoulder osteoarthritis & $14(16.3)$ \\
Shoulder muscle sprain & $1(1.2)$ \\
AC joint lesion & $37(43.0)$ \\
Calcified tendinitis & $2(2.3)$ \\
Unknown & $1(1.2)$ \\
\hline
\end{tabular}

SD Standard deviation, AC Acromioclavicular

\section{Internal consistency and test-retest reliability}

The Internal Consistency of our study was good, the Cronbach alpha value being 0.86 . The mean interval of the test-retest process was 12 days (S.D 5.4 days). The results of test-retest reliability showed excellent findings, with an ICC of 0.94 (95\% confidence interval 0.90,0.96; $p<0.01)$ for the total ASESp-CH score. The ICCs for each domain of ASESp-CH were all greater than 0.80 (Table 2).

\section{Construct validity}

The major domains of the ASESp-CH were significant correlated with the respective domains in the SF-36 $(p<0.01)$, except for the stability domain of ASESp-CH (Table 3). Moreover, the ASESp-CH total score was especially highly correlated (correlation $>0.7$ ) to the Physical Function, Role Limitation-Physical and Bodily Pain domain in the SF-36 $(p<0.001)$.

\section{Discussions}

As the patient-reported outcome measures (PROMs) are emphasized in clinical practice, the ASESp, a highly

Table 2 Test-retest reliability

\begin{tabular}{lll}
\hline ASESq domains & ICC $(\mathbf{9 5} \% \mathbf{C l})$ & $P$ value \\
\hline Pain & $0.83(0.75-0.89)$ & $<0.01$ \\
Stability & $0.85(0.78-0.90)$ & $<0.01$ \\
Daily activities & $0.96(0.93-0.97)$ & $<0.01$ \\
ASESq total scores & $0.94(0.90-0.96)$ & $<0.01$ \\
\hline
\end{tabular}


Table 3 Correlation between ASESq domains and the domains of the SF-36 questionnaire

\begin{tabular}{|c|c|c|c|c|c|c|c|c|}
\hline SF-36 domains & $\begin{array}{l}\text { Physical } \\
\text { function }\end{array}$ & $\begin{array}{l}\text { General } \\
\text { health }\end{array}$ & Vitality & $\begin{array}{l}\text { Mental } \\
\text { health }\end{array}$ & $\begin{array}{l}\text { Role limitations- } \\
\text { physical }\end{array}$ & $\begin{array}{l}\text { Role limitations- } \\
\text { emotional }\end{array}$ & $\begin{array}{l}\text { Social } \\
\text { functioning }\end{array}$ & $\begin{array}{l}\text { Bodily } \\
\text { pain }\end{array}$ \\
\hline \multicolumn{9}{|l|}{ ASESq domains } \\
\hline \multicolumn{9}{|l|}{ Pain } \\
\hline Correlation & $-0.568^{\mathrm{a}}$ & $-0.385^{\mathrm{a}}$ & $\overline{0.446^{\mathrm{a}}}$ & $-0.367^{\mathrm{a}}$ & $-0.674^{a}$ & $-0.366^{\mathrm{a}}$ & $-0.323^{a}$ & $-0.701^{a}$ \\
\hline $\begin{array}{l}\text { Significance (2- } \\
\text { tailed) }\end{array}$ & $<0.001$ & $<0.001$ & $\begin{array}{l}< \\
0.001\end{array}$ & $<0.001$ & $<0.001$ & $<0.001$ & 0.002 & $<0.001$ \\
\hline \multicolumn{9}{|l|}{ Stability } \\
\hline Correlation & 0.038 & -0.145 & -0.195 & -0.150 & 0.007 & 0.041 & 0.062 & -0.027 \\
\hline $\begin{array}{l}\text { Significance (2- } \\
\text { tailed) }\end{array}$ & 0.725 & 0.183 & 0.073 & 0.169 & 0.951 & 0.705 & 0.572 & 0.807 \\
\hline \multicolumn{9}{|l|}{ Activity } \\
\hline Correlation & $0.802^{a}$ & $0.386^{a}$ & $0.418^{a}$ & $0.412^{a}$ & $0.825^{a}$ & $0.547^{\mathrm{a}}$ & $0.485^{a}$ & $0.784^{a}$ \\
\hline $\begin{array}{l}\text { Significance (2- } \\
\text { tailed) }\end{array}$ & $<0.001$ & $<0.001$ & $\begin{array}{l}< \\
0.001\end{array}$ & $<0.001$ & $<0.001$ & $<0.001$ & $<0.001$ & $<0.001$ \\
\hline \multicolumn{9}{|l|}{ Total } \\
\hline Correlation & $0.760^{a}$ & $0.424^{\mathrm{a}}$ & $0.478^{a}$ & $0.431^{a}$ & $0.830^{a}$ & 0.507 & $0.447^{a}$ & $0.821^{\mathrm{a}}$ \\
\hline $\begin{array}{l}\text { Significance (2- } \\
\text { tailed) }\end{array}$ & $<0.001$ & $<0.001$ & $\begin{array}{l}< \\
0.001\end{array}$ & $<0.001$ & $<0.001$ & $<0.001$ & $<0.001$ & $<0.001$ \\
\hline
\end{tabular}

ASESq American Shoulder and Elbow Surgeons Standardized Shoulder Assessment Form, SF-36 36-Item Short Form Health Survey

${ }^{\text {a }}$ Correlation was significant at the alpha $<0.05$ level (2-tailed)

validated and reliable PROM, becomes a popular choice for measuring shoulder function $[4,5,8]$. Due to language and cultural diversity, ASESp had been translated into different languages across the world [4, 8, 10-15]. However, to our knowledge, a validated Chinese ASESp has not been proposed. The aim of the present study was to establish a Chinese version of ASESp, as known as the Chinese ASES questionnaire (ASESp-CH), to assess its reliability and validity. Our results showed that the proposed ASESp-CH was a validated and reliable tool for shoulder function assessment in this study.

During the translation processes, cross-cultural adaptions were necessary. Undoubtedly, some cross- cultural dissimilarities were found in the present study. Similar to the previous study [8], the major amendments were made due to cultural differences, such as the examples of pain control medicine, unit of weight, etc. Additionally, we also clearly define the description of "Manage Toileting" in question 4 of the ADL section as a "buttwiping" situation because of the cultural and language difference. After adequate adaption, no serious difficulties in realizing the statements were reported during pretesting.

Our study achieved a great result of internal consistency with a Cronbach alpha of 0.86. Cronbach Alpha values between 0.80 and 0.89 were regarded as good internal consistency [26]. Reviewing the previous adaptation studies $[4,8,10,12,13]$, most of the Cronbach Alpha values were in the range of 0.80
0.90. The Cronbach alpha value in our study was similar to the aforementioned studies $[4,8,10,12$, 13].

Regarding about test-retest reliability, an excellent ICC of 0.94 was found for the total ASESp-CH score. Compared with the prior studies $[4,8,10,12,13]$, test-retest reliability in the present study was relatively great, even the mean test-retest interval reached 12 days. The finding was impressive, as the previous studies $[8,11]$ suggested that a longer test-retest interval could reduce memory bias yet result in lower ICC. Therefore, the ASESp-CH could be considered a well-translated and well-constructed questionnaire that could be fullyunderstand without too many interpretation errors.

The present study also evaluated the construct validity for the ASESp-CH. We compared the domains of the ASESp-CH with the domains of the validated and translated Chinese instruments Short-Form 36 questionnaire (SF-36) [24, 25]. A good construct validity was found for the domains of ASESp-CH to the corresponding domains in SF-36, except for the stability domain. It is exciting to find that the ASESp-CH was significantly correlated to all of the domains of SF-36, although the previous studies of other language versions were only correlated to some SF-36 domains $[8,10]$. Furthermore, the ASESp-CH was highly correlated to physical functioning, role limitation-physical and bodily pain domains of SF-36, suggesting the ASESp-CH to be an appropriate tool for clinical evaluation. 
Unfortunately, the domain of stability was not significant correlated to SF-36 in our study. Although the previous study [8] reported significant correlation between the stability and some SF-36 domains, only modestly correlation was identified. The possible reason for the above findings was that the clinical definition of instability could not be fully understood by the patient without instability. From the clinical observation during the study, some patients have questions regarding the definition of instability, even though an example, sensation of shoulder dislocation, has been given in the questionaire. To minimized the misunderstanding in this section, we suggested that adequate explanation of shoulder instability should be given to the patients before filling out the questionaires.

\section{Limitations}

The present study had some limitations. First, there was no final consensus on the way of cross-cultural adaptation and validation. The present study selected the most respected guidelines, which were also used by previous ASESp adaptation and validation studies. Second, a large proportion of senior elders with age above 75 were not able to speak, read, or even write in Chinese. The patients were unfortunately excluded in this study, which possibly leading to a selection bias and the compromised reliability in elderly population. Third, although the sample size of our study considered satisfied, however, the ratio of gender was not well-balanced as the aforementioned studies. Lastly, Chinese is a commonly used language in several countries and regions, including Taiwan, China, Hong Kong, Macau, South-east Asia etc. Care should be taken while using the translated questionnaire in our study because the cultural difference was noticeable and some brand names of medicine were not identical among different regions. Therefore, minor revision of the current questionnaire is suggested before application in different regions.

\section{Conclusions}

The Chinese version of ASES questionnaire is a highly validated and reliable tool for shoulder disorder assessment.

\section{Abbreviations}

ASESp: American Shoulder and Elbow Surgeons Standardized Shoulder Assessment Form; ASESp-CH: Chinese version of ASESp; ICC: Intraclass correlation; SF-36: 36-Item Short Form Health Survey; ADL: Activities of daily living; QOL: Quality of life; VAS: Visual analog scale; PROM: Patient-reported outcome measures

\section{Supplementary Information}

The online version contains supplementary material available at https://doi. org/10.1186/s12891-021-04255-z.

Additional file 1. ASESp Questionnaire.

\section{Acknowledgements}

We thank Skeleton Materials and Bio-compatibility Core Lab, Research Center of Clinical Medicine, National Cheng Kung University Hospital, Tainan, Taiwan for assistance with this project.

\section{Authors' contributions}

C-K H and K-L H contributed to the methodology. T-A T, C-K H, I C, and K-L $\mathrm{H}$ contributed to the methodology. T-A T and C-K H drafted the manuscript. F-C K and W-R S contributed to critical review of the manuscript. K-L H supervised the study. All authors read and approved the manuscript.

\section{Funding}

This study was funded by National Cheng Kung University Hospital, Tainan, Taiwan (NCKUH-1 1002039).

\section{Availability of data and materials}

The datasets used and analyzed during the current study are available from the corresponding author on reasonable request.

\section{Declarations}

\section{Ethics approval and consent to participate}

The study was approved by the Institutional Review Board of National Cheng Kung University Hospital (A-ER-109-163). All participants completed the written informed consents.

\section{Consent for publication}

Not applicable.

\section{Competing interests}

The authors declared that there was no conflict of interest.

\section{Author details}

${ }^{1}$ National Cheng Kung University Hospital, College of Medicine, National Cheng Kung University, No.138, Sheng-Li Road, North Dist., Tainan City 70428, Taiwan. ${ }^{2}$ Department of Orthopaedic Surgery, National Cheng Kung University Hospital, College of Medicine, National Cheng Kung University, No.138, Sheng-Li Road, North Dist., Tainan City 70428, Taiwan. ${ }^{3}$ Department of Pharmacy, College of Medicine, National Cheng Kung University, No. 1 University Road, East Dist., Tainan City 70101, Taiwan. ${ }^{4}$ Department of Biomedical Engineering, National Cheng Kung University, No.138, Sheng-Li Road, North Dist., Tainan City 70428, Taiwan. ${ }^{5}$ Skeleton Materials and Bio-compatibility Core Lab, Research Center of Clinical Medicine, National Cheng Kung University Hospital, No.138, Sheng-Li Road, North Dist., Tainan City 70428, Taiwan.

Received: 12 January 2021 Accepted: 13 April 2021

Published online: 24 April 2021

\section{References}

1. Linaker $\mathrm{CH}$, Walker-Bone K. Shoulder disorders and occupation. Best Pract Res Clin Rheumatol. 2015;29(3):405-23.

2. Luime JJ, Koes BW, Hendriksen IJ, Burdorf A, Verhagen AP, Miedema HS, et al. Prevalence and incidence of shoulder pain in the general population; a systematic review. Scand J Rheumatol. 2004;33(2):73-81.

3. Sarquis LM, Coggon D, Ntani G, Walker-Bone K, Palmer KT, Felli VE, et al. Classification of neck/shoulder pain in epidemiological research: a comparison of personal and occupational characteristics, disability, and prognosis among 12,195 workers from 18 countries. Pain. 2016;157(5):1028-36.

4. Policastro PO, Pierobon A, Perez J, Novoa GA, Calvo Delfino M, Sajfar ME, et al. Cross-cultural adaptation and validation of the Argentine "American shoulder and elbow surgeons, patient self-report section" questionnaire. Musculoskelet Sci Pract. 2019;43:37-44.

5. Schmidt S, Ferrer M, Gonzalez M, Gonzalez N, Valderas JM, Alonso J, et al. Evaluation of shoulder-specific patient-reported outcome measures: a systematic and standardized comparison of available evidence. J Shoulder Elb Surg. 2014;23(3):434-44.

6. Biz C, Crimì A, Belluzzi E, Maschio N, Baracco R, Volpin A, et al. Conservative versus surgical management of elbow medial ulnar collateral ligament injury: a systematic review. Orthop Surg. 2019;11(6):974-84. 
7. Poolman RW, Swiontkowski MF, Fairbank JC, Schemitsch EH, Sprague S, de Vet HC. Outcome instruments: rationale for their use. J Bone Joint Surg Am. 2009;91(Suppl 3):41-9.

8. Felsch QTM, Sievert P, Schotanus MGM, Jansen EJP. The Dutch version of the American shoulder and elbow surgeons standardized shoulder assessment form is a reliable and valid questionnaire for shoulder problems. JSES Open Access. 2019;3(3):213-8.

9. Michener LA, McClure PW, Sennett BJ. American shoulder and elbow surgeons standardized shoulder assessment form, patient self-report section: reliability, validity, and responsiveness. J Shoulder Elb Surg. 2002; 11(6):587-94.

10. Celik D, Atalar AC, Demirhan M, Dirican A. Translation, cultural adaptation, validity and reliability of the Turkish ASES questionnaire. Knee Surg Sports Traumatol Arthrosc. 2013;21(9):2184-9.

11. Goldhahn J, Angst F, Drerup S, Pap G, Simmen BR, Mannion AF. Lessons learned during the cross-cultural adaptation of the American shoulder and elbow surgeons shoulder form into German. J Shoulder Elb Surg. 2008;17(2): 248-54

12. Padua R, Padua L, Ceccarelli E, Bondi R, Alviti F, Castagna A. Italian version of ASES questionnaire for shoulder assessment: cross-cultural adaptation and validation. Musculoskelet Surg. 2010;94(Suppl 1):S85-90.

13. Piitulainen K, Paloneva J, Ylinen J, Kautiainen $\mathrm{H}$, Hakkinen A. Reliability and validity of the Finnish version of the American shoulder and elbow surgeons standardized shoulder assessment form, patient self-report section. BMC Musculoskelet Disord. 2014;15:272.

14. Vrotsou K, Cuellar R, Silio F, Rodriquez MA, Garay D, Busto G, et al. Patient self-report section of the ASES questionnaire: a Spanish validation study using classical test theory and the Rasch model. Health Qual Life Outcomes. 2016;14(1):147

15. Yahia A, Guermazi M, Khmekhem M, Ghroubi S, Ayedi K, Elleuch MH. Translation into Arabic and validation of the ASES index in assessment of shoulder disabilities. Ann Phys Rehabil Med. 2011;54(2):59-72.

16. Beaton DE, Bombardier C, Guillemin F, Ferraz MB. Guidelines for the process of cross-cultural adaptation of self-report measures. Spine (Phila Pa 1976). 2000;25(24):3186-91.

17. Bejer A, Szczepanik M, Plocki J, Szymczyk D, Kulczyk M, Pop T. Translation, cross-cultural adaptation and validation of the polish version of the Oxford shoulder score in patients undergoing arthroscopic rotator cuff repair. Health Qual Life Outcomes. 2019;17(1):191.

18. Gadisa DA, Gebremariam ET, Ali GY. Reliability and validity of Amharic version of EORTC QLQ-C30 and QLQ-BR23 modules for assessing healthrelated quality of life among breast cancer patients in Ethiopia. Health Qual Life Outcomes. 2019;17(1):182.

19. Josipovic P, Moharic M, Salamon D. Translation, cross-cultural adaptation and validation of the Slovenian version of Harris hip score. Health Qual Life Outcomes. 2020;18(1):335.

20. Zmnako SSF, Chalabi YI. Cross-cultural adaptation, reliability, and validity of the Vertigo symptom scale-short form in the central Kurdish dialect. Health Qual Life Outcomes. 2019;17(1):125.

21. Tavakol M, Dennick R. Making sense of Cronbach's alpha. Int J Med Educ. 2011;2:53-5.

22. Koo TK, Li MY. A guideline of selecting and reporting Intraclass correlation coefficients for reliability research. J Chiropr Med. 2016;15(2):155-63.

23. Ware JE Jr. SF-36 health survey update. Spine (Phila Pa 1976). 2000;25(24): 3130-9.

24. Lu JR, Tseng HM, Tsai YJ. Assessment of health-related quality of life in Taiwan (I): development and psychometric testing of SF-36 Taiwan version. Taiwan J Public Health. 2003;22(6):501-11.

25. Tseng HM, Lu JR, Tsai YJ. Assessment of health-related quality of life in Taiwan (II): norming and validation of SF-36 Taiwan version. Taiwan J Public Health. 2003;22(6):512-8

26. Cicchetti DV. Guidelines, criteria, and rules of thumb for evaluating normed and standardized assessment instruments in psychology. Psychol Assess. 1994;6:284-90.

\section{Publisher's Note}

Springer Nature remains neutral with regard to jurisdictional claims in published maps and institutional affiliations.

\section{Ready to submit your research? Choose BMC and benefit from:}

- fast, convenient online submission

- thorough peer review by experienced researchers in your field

- rapid publication on acceptance

- support for research data, including large and complex data types

- gold Open Access which fosters wider collaboration and increased citations

- maximum visibility for your research: over $100 \mathrm{M}$ website views per year

At BMC, research is always in progress.

Learn more biomedcentral.com/submissions 